\title{
Tangence
}

\section{L'hétérogénéité : une passion " postmoderne » (?)}

\section{Claude-Maurice Gagnon}

Numéro 39, mars 1993

La fiction postmoderne

URI : https://id.erudit.org/iderudit/025753ar

DOI : https://doi.org/10.7202/025753ar

Aller au sommaire du numéro

Éditeur(s)

Tangence

ISSN

0226-9554 (imprimé)

1710-0305 (numérique)

Découvrir la revue

Citer cet article

Gagnon, C.-M. (1993). L'hétérogénéité : une passion « postmoderne » (?).

Tangence, (39), 62-75. https://doi.org/10.7202/025753ar d'utilisation que vous pouvez consulter en ligne.

https://apropos.erudit.org/fr/usagers/politique-dutilisation/ 


\section{L’hétérogénéité: une passion " postmoderne " (?)1 Claude-Maurice Gagnon}

Dans le cadre de ce dossier intitulé La fiction postmoderne, je propose une réflexion sur le phénomène de l'hétérérogénéité qui représente, depuis l'entrée en scène des avant-gardes, l'une des deux tendances majeures des productions artistiques visuelles du $\mathrm{xX}^{\mathrm{c}}$ siècle et qui se perpétue dans l'art actuel, québécois notamment.

Autrement dit, il s'agit de montrer, à partir d'exemples pertinents, issus des productions de l'avant- et de la trans-avantgarde $^{2}$ - termes ne correspondant plus aux classifications taxinomiques dictées par les lois strictes de la spécificité - comment les artistes qui prennent part à ce bouleversement idéologique imposent une rupture dans l'ordre du langage, déstabilisant ainsi tous les acquis et préjugés, de goût et de talent, ancrés dans la tradition des Beaux-Arts; comment aussi ils participent à la constitution d'un nouveau code ouvert à tous les métissages, intégrant, outre le rapport transdisciplinaire aux divers champs d'intervention des arts visuels et de la culture littéraire, le rapport transculturel au non-art et à la culture populaire.

1 L'essentiel de cet article a été présenté, en novembre 1992, à l'American Council For Quebec Studies, dans le colloque de Marie Carani (Département d'histoire, Université Laval), intitulé Visual Semiotics and Contemporary Quebec Art History. L'auteur est boursier du Conseil des arts du Canada

2 Véhiculée par le critique Achille Bonito-Oliva, la notion de strans-avantgarde* concerne d'abord les productions artistiques visuelles réalisées, dès le début des années quatre-vingt, par les artistes italiens Cucchi, Paladino, De Maria et Clemente. Elle signifie également : un art de transition, de passage, qui n'oublie pas les leçons des avant-gardes, mais qui s'ouvre sur d'autres options culturelles, les "traditions nationales" en dehors de cet internationalisme obligé qui avait caractérisé d'autres mouvements*, comme il l'explique dans son article *Ainsi l'état de l'art (et de la critique aussi) ", New Art International, avril-mai 1990, p. 19-31. Or, c'est précisément dans ce sens que je reprends cette notion et que je l'applique à l'art actuel québécois. Je renvoie le lecteur à mon analyse du texte de Bonito-Oliva cité plus haut: * L'histoire, un matériau: Greenberg, Bonito-Oliva, Scarpetta ", Espace (sculpture), n 18, hiver 1992, p. 20-25. 
Partant de là, j'envisage le phénomène de l'hétérogénéité artistique comme la base d'une révolution trans-linguistique ayant pris forme sous le modernisme et j'associe les constructions artistiques impures que cette révolution engendre à l'attitude "postmoderne". Ainsi, la notion imprécise de "postmoderne" ne connoterait pas ici un moment historique après le modernisme, mais révélerait, à l'intérieur même du modernisme, un de ses axes limitrophes, soit celui de l'hétérogénéité, l'autre étant celui de la spécificité.

Dans cette perspective, trois corpus retiennent mon attention: du côté des productions de l'avant-garde, les ready-made de Duchamp et les collages de Max Ernst et, du côté de la transavant-garde québécoise, les "tableaux poétiques" de Nathalie Caron. Pour l'essentiel, cette analyse cherche à questionner comment s'ordonnent, s'agencent, se bricolent et/ou s'édifient, dans la logique "postmoderne" du modernisme, les dimensions syntaxiques, sémantiques et symboliques de ces constructions artistiques profondément hétérogènes.

\section{Le jeu iconoclaste du ready-made}

La surprise est le grand ressort du nouveau. C'est par la surprise, par la place importante qu'il fait à la surprise, que l'esprit nouveau se distingue ${ }^{3}$.

L'incompréhension et le refus du tableau de Duchamp intitulé $N u$ descendant un escalier (1912) au Salon des Indépendants amène cet artiste à se détourner de la peinture vers l'objet et à produire, par ce passage, une transformation idéologique de la définition même de l'art. Ce faisant, il réalise, en 1913, son premier ready-made aidé, la Roue de bicyclette, tandis qu'il conçoit, en 1914, son premier ready-made, Porte-bouteilles, et son premier ready-made rectifié, Pharmacie, causant une seconde catastrophe ${ }^{4}$, également historique, et obligeant le

3 Guillaume Apollinaire, L'esprit nouveau et les poètes, Paris, Imprimerie J. Haumont, 1946.

4 Je reprends une autre expression du critique italien Achille Bonito-Oliva, lequel s'inspirant de la philosophie de Nietzsche (pour qui l'acte de 
spectateur à rompre avec toute perception naturalisante de l'art, telle, par exemple, celle qui consiste à associer uniquement la production artistique à la fabrication manuelle d'un objet, tandis que celle-ci pourrait découler d'une activité de choix, comme il l'a expliqué dans son article "The Richard Mutt Case", à propos de son ready-made aidé, Fontaine, refusé à la Société des artistes indépendants (New York, 1917):

Que Mr. Mutt [pseudonyme de Duchampl ait fait la Fontaine de ses propres mains ou non n'a pas d'importance. Il l'a choisie. Il a pris un, article courant, l'a placé de telle sorte que sa signification utilitaire disparaisse sous le nouveau titre et le nouveau point de vue - il a créé pour cet objet une nouvelle idée 5 .

Dans ce sens, il me paraît que le choix du ready-made en tant que procédé d'appropriation et de citation correspond, toujours déjà et à la fois, à un détournement de l'objet fonctionnel dans l'espace réel et symbolique, et à une remise en question de la fonction sociale et linguistique de l'art. Par le transfert systématique de l'objet arbitraire de son contexte habituel et son isolement symbolique dans un contexte autre que celui de l'art l'art peut-il signifier un lieu autre que celui de l'autre? -, Duchamp met intentionnellement l'accent sur la dimension structurale de l'objet et sur ses propriétés intrinsèques, tandis que la proposition littéraire qui l'accompagne, sous la forme ironique du calembour (tautologie, métaphore, analogie, etc.), contribue, dans ce rapport trans-linguistique, à déstabiliser, chez le spectateur, la perception même de l'objet d'art. Autrement dit, puisque le ready-made affirme exemplairement son état de "modèle réduit " ${ }^{-}$- il expose sa structure - sa réalité structurale se

créer est guidé par une intention de destruction) comprend généralement l'art comme un *geste catastrophe antisocial». Achille BonitoOliva, op. cit.

5 Marcel Duchamp, The Richard Mutt Case., New York, The Blind Man/P.B.T., n' 2, mai 1917, p. 5; cité par Arturo Schwarz, Marcel Duchamp: la mariée mise à nu cbez Marcel Ducbamp, même, Paris, Éd. G. Fall, Bibli. Opus, 1974, p. 60.

6 Pour la notion de "modèle réduit", je retourne le lecteur à l'ouvrage de Claude Lévi-Strauss, La pensée sauvage, Paris, Plon, 1962, ainsi qu'à mon article - L'apport de Claude Lévi-Strauss et de Julia Kristeva face à l'élaboration d'une sémiotique de l'hétérégonéité artistique dans le champ de l'histoire de l'art., Marie Carani (et autres), De l'bistoire de l'art à la sémiotique visuelle, Québec, Éd. Du Septentrion/CELAT (Université Laval), 1992. 
trouve pervertie par la "fission sémantique "7 qu'entraînent sa déet sa re-contextualisation, ainsi que par son rapport polysémique à l'énoncé linguistique qui lui sert poétiquement de titre ${ }^{8}$.

C'est du moins ce dont témoignent, entre autres, la pelle à neige suspendue dans l'espace, intitulée In Advance of the Broken Arm (En avance du bras cassé) (1915), et le Peigne en acier portant sur la tranche de son côté supérieur l'inscription suivante: 3 ou 4 gouttes de hauteur n'ont rien à faire avec la sauvagerie". Ainsi, ce qui fait que le ready-made est un objet d'art ce n'est pas sa construction matérielle - puisque par définition il est déjà construit - mais plutôt le choix et le déplacement de cet objet arbitraire dans l'espace artistique d'exposition, ainsi que les subtiles transformations syntaxiques que l'artiste lui fait quelquefois subir. En le théâtralisant dans ce nouvel environnement, par le biais de la citation qui fonde son nomadisme et en lui donnant un titre qui déchire et/ou fracture sa dimension sémantique/symbolique originelle tout en lui conférant une signification polysémique qui use de la métaphore, Duchamp fait du ready-made non pas un signe, mais un hypersigne, qui ne cherche pas à communiquer autre chose qu'un métadiscours sur l'hétérogénéité de l'environnement social, culturel et artistique, de même que sur l'hétérogénéité pulsionnelle du sujet qui choisit l'objet en brisant tous les codes de la communication même. Pour retourner au langage kristévéen, la conceptualisation du ready-made se manifeste comme "re-jet" 9 ,

7 Claude Lévi-Strauss, dans Georges Charbonnier, Entretiens avec Claude Lévi-Strauss, Paris, UGE, coll. * 10/18*, 1969, p. 112.

8 J'abonde ici dans le sens de Claude Lévi-Strauss qui mentionne que: - Dans les ready-mades [...] ce sont les "phrases" faites avec des objets qui ont un sens, et non pas l'objet seul, quoi qu'on ait voulu faire ou dire. C'est un objet dans un contexte d'objets et, bien entendu, on pourrait concevoir à la limite qu'une civilisation qui se trouverait entièrement prisonnière $[. .$.$] de son univers technique et matériel arrive à$ le disposer de plusieurs manières [...] qui seraient des manières gratuites et artistiques, et que la différence entre les deux ne tienne qu'aux arrangements. Un coquillage n'est pas la même chose dans une galerie du Muséum d'Histoire Naturelle ou sur la table de l'amateur de curiosités. Claude Lévi-Strauss, dans Georges Charbonnier, op. cit., p. 115.

9 Pour un développement de la notion de * re-jet *, j'indique au lecteur l'essai de sémanalyse de Julia Kristeva, La Révolution du langage poétique; l'avant-garde à la fin du XIX siècle: Lautréamont et Mallarmé, Paris, Seuil, 1974, p. 155; ainsi que mon article déjà mentionné à la note 4. 
66

c'est-à-dire comme un geste de rupture procédant par déplacement et condensation. Celui-ci est paradoxalement régi par une stratégie de réunification des codes du majeur et du mineur, du langage visuel et verbal, marquant ainsi la suite de l'histoire de l'art.

D'une part, par sa critique épistémologique de la représentation artistique traditionnelle - laquelle avait toujours reposé sur l'intention naturalisante de faire voir des reproductions mimétiques et élogieuses du "réel " -, Duchamp impose et oppose la matérialité de l'objet arbitraire qu'il rend dysfonctionnel en le présentant poétiquement comme objet d'art. D'autre part, par ce geste iconoclaste qui participe d'une perversion suprême, Duchamp pose donc une théorie de l'objet d'art, c'est-à-dire une théorie de l'art en tant qu'objet, qui implique et sous-tend, au delà de la représentation, une théorie de la présentation du dispositif artistique et de sa théâtralisation dans l'espace réel et symbolique.

Bref, il s'agit d'une déstabilisation totale des acquis et d'une relecture sémiotique de l'artefact menées par la passion du travestissement et/ou du pervertissement du sens, nécessitant une (re)création des codes linguistiques, leur (re)modélisation. Incontestablement, il y a là rupture incontournable: "catastrophe" opérée par les stratégies "postmodernes" de l'hétérogénéité que sont l'appropriation, le nomadisme, le transcodage, le jeu, le plaisir de jouer en ironisant et en détournant les choses et le sens des choses au profit d'une profusion de sens et de choses, dans la logique passionnelle du second degré. Hétérogénéité maximale que Lautréamont avait déjà actualisée dans sa formulation poétique, maintenant historique: "Beau comme la rencontre fortuite sur la table de dissection d'une machine à coudre et d'un parapluie! " ${ }^{10}$.

\section{Max Ernst à l'avant-scène du collage}

Dans son texte "La peinture au défi " publié en 1930 - soit dix-neuf ans après les premiers collages de Braque et de Picasso - , le poète Aragon propose une lecture critique inaugurale du collage, prouvant ainsi le retard de l'histoire de l'art sur l'actualité et l'innovation artistiques, ici l'hétérogénéité: 
Il est curieux, affirme Aragon, que presque personne n'ait semblé prendre garde à une occupation singulière, dont les conséquences ne sont pas encore toutes appréciables, à laquelle certains hommes se sont livrés ces temps-ci d'une façon systématique qui rappelle plus les opérations de la magie que celles de la peinture. Outre qu'elle met en question la personnalité, le talent, la propriété artistique et toutes sortes d'idées qui chauffaient sans méfiance leurs pieds tranquilles dans les cervelles crétinisées. Je veux parler de ce qu'on appelle pour simplifier le collage, bien que l'emploi de la colle ne soit qu'une des caractéristiques de cette opération, et même pas une caractéristique essentielle. Sans doute que ce sujet avait en soi quelque chose qui effrayait les esprits, car, dans toute la critique volumineuse consacrée depuis sa naissance au cubisme, par exemple, on ne trouve que quelques mots superficiels pour noter l'existence des papiers collés, comme on disait d'abord, pour désigner cette première apparition du collage par laquelle se révèle l'inquiétude de Braque et de Picasso dès 1911. Il faut dès maintenant préciser que le collage tel qu'on l'entend aujourd'hui est quelque chose d'entièrement différent du papier collé du cubisme. Mais celui-ci posait déjà certaines questions que celui-là pose encore. Il est significatif de noter cette carence critique, vis-à-vis d'une activité qui absorba une bonne part de la pensée des plus admirés d'entre les peintres d'une génération; les quelques mots que l'on en a donné comme excuse plus que comme explication ne suffisent même pas à établir l'interprétation plastique qu'on était en droit d'attendre, à une époque où les considérations de cet ordre étaient à la mode parmi les commentateurs ${ }^{11}$.

Aragon distingue deux catégories de collage. La première, beaucoup plus sage que la seconde, reste fixée à la spécificité de la peinture - abstraite. Si elle intègre des éléments extrapicturaux, tels des papiers, des textiles, des cartons découpés géométriquement ou des mots et des chiffres, ceux-ci sont collés sur la surface bidimensionnelle de l'espace pictural et l'aspect critique de cette manière tend, tout au plus, à enrichir les limites chromatiques de la "palette " et à permettre l'intégration de nouveaux effets matériologiques de texture. Cette catégorie renvoie aux collages cubistes. Eu égard à l'hétérogénéité, la plus pertinente de ces deux catégories est gérée par un ardent désir de contaminer la pratique picturale et travaille au débordement du champ de la peinture; elle inclut l'élément collé spécifiquement

11 Louis Aragon, *Ia peinture au défi *, Les collages, Paris, Hermann, 1980, p. 44-45. 
pour mettre l'accent sur "la représentation de l'objet "12 et ne valorise plus exclusivement l'abstraction: au contraire, elle cherche à décoller de cette problématique puriste. Cette catégorie concerne les collages dadaïstes et surréalistes, principalement. Ainsi, les collages "postcubistes", issus de cette seconde catégorie, se préoccupent davantage de "l'exprimé "que de la "manière de l'exprimé "13, abandonnent la question du goût et du beau, optent pour celle du choix, se détachent de la conception de l'art pour l'art que la spécificité commande. Ce qu'ils visent à tout prix, c'est de revenir à la magie de l'art, à sa nécessité symbolique, à sa fonction rituelle, en mélangeant les codes de l'expression artistique, en abolissant toutes frontières, celles de la peinture pour commencer:

Le principe du collage admis, pose Aragon, les peintres avaient passé sans en rien savoir de la magie blanche à la magie noire. Il était trop tard pour reculer. On peut imaginer le temps où les problèmes de la peinture, et par exemple ceux qui ont fait le succès du cézannisme, sembleront aussi étranges, aussi anciens que les tourments prosodiques des poètes peuvent dès maintenant paraître. On peut imaginer le temps où les peintres qui ne broyent déjà plus eux-mêmes leurs couleurs trouveront enfantin et indigne d'étaler eux-mêmes la peinture, et ne reconnaîtront plus à cette touche personnelle qui fait aujourd'hui encore la valeur de leurs tableaux que l'intérêt documentaire du manuscrit, de l'autographe. On peut imaginer le temps où les peintres ne feront même plus étaler par d'autres la couleur, ne dessineront même plus. Le collage nous donne un avant-goût de ce temps-là ${ }^{14}$.

Le collage moderne $[. .$.$] requiert [. .$.$] notre attention pour ce$ qu'il a de concerté, d'absolument opposable à la peinture, audelà de la peinture. Pour ce qu'il représente de possibilité humaine. Pour ce qu'il substitue à un art avili un mode d'expression d'une force et d'une portée inconnues. Pour ce qu'il restitue son sens véritable à la vieille démarche picturale, en empêchant le peintre de s'adonner au narcissisme, à l'art pour l'art, en le ramenant aux pratiques magiques qui sont l'origine et la justification des représentations plastiques, défendues par plusieurs religions ${ }^{15}$.

12 Ibid., p. 47.

13 Ibid., p. 47

14 Ibid., p. 48-51.

15 Ibid., p. 58. 
Cela dit, et dans la mesure où il privilégie le surréalisme plutôt que le réalisme matérialiste du cubisme, Aragon attribue à Max Ernst, plutôt qu'à Picasso, une place de choix dans le développement du collage comme genre hétérogène: il rappelle qu'après les premiers papiers collés de Braque et de Picasso, la pertinence de la contribution de Max Ernst réside dans ses collages photographiques et ses collages d'illustrations, dont les procédés furent imités massivement par les photomontages des artistes dadaïstes allemands (Hausmann, Hoch, Grosz, etc.) et ceux des constructivistes russes (Rodtchenko, Lissitsky, Moholy-Nagy, etc.), lesquels les concevaient à des fins de propagande et d'action politique, contrairement au contenu poétique qui se dégageait de ceux que produisait Ernst et dont la réalisation participait déjà de l'écriture automatiste.

Dépassant, sur le plan syntaxique, le système des papiers collés, les collages de Max Ernst se complexifiaient, en métissant "l'élément photographique collé dans un dessin ou une peinture; l'élément dessiné ou peint surajouté à une photographie; l'image découpée et incorporée à un tableau ou à une autre image; la photographie pure et simple d'un arrangement d'objets rendu incompréhensible par la photographie "16. D'autre part, sur le plan sémantique/symbolique, le collage ernstien se distingue du collage cubiste où l'objet cité "procède d'une intention réaliste ou, du moins, d'une volonté de confronter les signes iconiques aux signes de la réalité quotidienne" ou sociologique, comme le souligne Henri Béhar dans son essai "La saveur du réel "17. Chez Ernst, puisque les éléments collés sont des fragments dessinés ou photographiés élaborant déjà, dans un autre contexte linguistique, un métadiscours sur le "réel", l'enjeu de la représentation du collage participe directement, en tant que "re-jet", de la stratégie poétique surréaliste où ce qui est montré témoigne immédiatement de la réalité intérieure du sujet et d'un projet de déréalisation et de dénaturalisation de la réalité extérieure.

Ainsi, dans le collage ernstien, la construction visuelle de la représentation, qui s'édifie par le biais d'une rhétorique de l'illusion et de la contradiction, vise la confrontation symbolique des motifs iconiques, leur dépaysement, par la destruction des

16 Ibrid., p. 65.

17 Henri Béhar, -La saveur du réel *, Europe (Cubisme et littérature), $\mathrm{n}^{\mathrm{s}}$ 638-639, juin-juillet 1982, p. 101. 
repères logiques. Oiseaux, plumes, jupes gonflées, fragments de corps, insectes, animaux, vampires, personnages androgynes, personnages en chute libre dans l'espace constituent les motifs récurrents qui concourent à transposer l'hétérogénéité sémantique/symbolique de l'image rêvée dans la construction syntaxique et hétérogène du collage. L'illusion de la représentation, sa dimension de simulacre, est accrue par le gommage des interventions manuelles: aucune trace de colle n'est apparente, ni ne paraît dans l'espace liant deux motifs, celui-ci étant dissimulé par le dessin. Si ces collages ne sont plus de la peinture, ils ne cessent pourtant de parler d'elle, si bien que dans plusieurs cas toutes les surfaces fragmentées de la représentation ont été retouchées par les moyens mêmes de la peinture; dans ce sens, il y a une volonté de troubler la perception; de confondre le regardeur sur la matérialité même des jeux de langues assimilés; d'ironiser la hiérarchie des codes dans l'intention "postmoderne" de la déjouer, juste pour le plaisir; de suggérer l'idée du faux, d'une fausse peinture, du "mineur", bref, de foutre en l'air toute la tradition, faisant ainsi de l'art du collage le lieu d'un théâtre métaphorique où se manifeste le "conflit des éléments disparates "18, et ce, dans la plus grande insolence. Partant de là, ce qu'il s'agit de reproduire, et sans laisser de traces du faire artistique qui briseraient l'effet magique recherché, c'est, ni plus ni moins, L'intêrieur de la vue, comme le suggère le titre d'un collage de Max Ernst. Conséquemment, le collage ernstien se définit comme une mise en scène de l'inconscient opérant par le biais de projections fantasmatiques et par des stratégies de détournements et de perversions sémantiques/symboliques du signe qui participent de la remontée du refoulé. Ce collage est spatialement organisé par une syntaxe hétérogène qui cherche à faire apparaître du merveilleux, ce qu'Aragon associe aussi à la surréalité du miracle:

Le miracle est un désordre inattendu, une disproportion surprenante. Et c'est à cet égard qu'il est la négation du réel, et qu'il devient une fois accepté le miracle, la conciliation du réel et du merveilleux. Le nouveau rapport établi est la surréalité, mille fois définie, et toujours différemment définissable, cette ligne réelle qui relie toutes les images virtuelles qui nous entourent ${ }^{19}$. 


\section{Nathalie Caron : la poésie visuelle de quatre mouchoirs}

Dans son essai Les mots dans la peinture ${ }^{20}$, Michel Butor s'intéresse aux correspondances qui lient, dans la production artistique occidentale - depuis le Moyen-Âge jusqu'à l'époque contemporaine - le texte et la peinture et lit les textes dans les peintures ${ }^{21}$, montrant que ces deux types de productions artistiques ne sont pas à lire comme des productions parallèles, mais plutôt qu'elles s'interpénètrent dans la logique hétérogène des correspondances. Du point de vue d'une sémiotique de l'hétérogénéité artistique, l'intérêt majeur de l'essai de Butor réside dans son appréhension du mot comme dessin et image, c'est-à-dire comme signe linguistique relevant originellement du langage verbal. Une fois déplacé de ce contexte d'appartenance, celui-ci devient, dans le système des arts visuels, signe visuel et communique du sens dans une perspective iconique. Butor reprend la même idée dans son article "Travailler avec les peintres " où il relate son expérience d'illustration d'œuvres picturales, par le biais du mot, dans le livre illustré:

À partir du moment où l'œuvre était faite, j’essayais pourtant de la détacher; ces textes qui en principe ne pouvaient pas se détacher des images qui les accompagnaient, pour réussir à les détacher, il a fallu souvent que je les transforme et que j'en transforme en particulier la présentation, c'est-à-dire qu'il a fallu que je fasse de la peinture avec mes textes. [...] Ainsi, poursuit-il, les peintres nous enseignent beaucoup sur le pouvoir des mots et sur la façon dont le sens des mots est transformé d'abord par le décor dans lequel on les met, et puis par les relations plastiques qu'il peut y avoir entre ces mots. Les peintres nous rappellent perpétuellement que l'écriture c'est du dessin qui est juxtaposé à quelque chose de complètement différent, mais qu'en réalité nous avons deux sortes de dessins qui sont mis en présence. L'écriture c'est un dessin qui a des propriétés particulières, qui a notamment des vecteurs (les mots nous obligent à les parcourir dans un certain sens), des forces pour nous irrésistibles ${ }^{22}$.

20 Michel Butor, Les mots dans la peinture, Genève, Éd. Albert Skira, coll. * Les Sentiers de la création *, 1969.

21 Le titre, la signature, la légende, les inscriptions au verso des oeuvres, la présence de textes d'armoiries, le nom des saints souvent représenté en auréole au-dessus de la tête, les représentations de lettres, de livres, le collage d'imprimés et de lettres, la figuration de hiéroglyphes et de dessins-poèmes, etc.

22 Michel Butor, -Travailler avec les peintres", Arts et littérature, collectif dirigé par Hans-Jürgen Greif, Québec, Éd. Nuit Blanche, 1987, p. 26-27. 


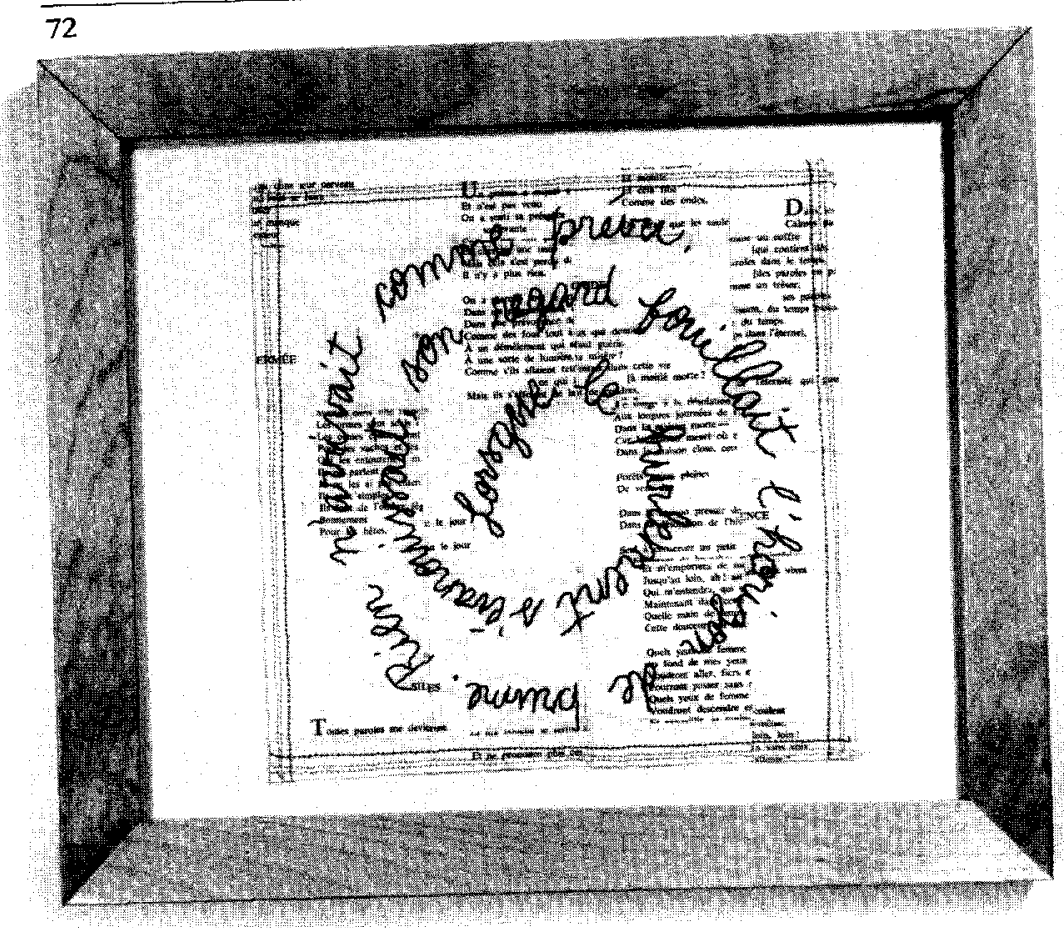

Or, ce déplacement du signe linguistique verbal dans le système linguistique visuel de la peinture ne participerait-il pas, par analogie, de la même logique de détournement que soustend l'intégration de l'objet arbitraire dans le ready-made ou le transcodage du document imprimé et/ou photographique dans le collage? N'y a-t-il pas, dans chacun des cas, une transformation de la perception, de la fonction et de la signification du signe décontextualisé, due à son passage translinguistique dans un autre système linguistique?

Dans ce sens, la pratique artistique hétérogène de Nathalie Caron, qui s'inscrit dans la contemporanéité de la trans-avantgarde québécoise et/ou de l'art québécois en général, s'inspire tant du ready-made que du collage et de la culture littéraire qu'elle assimile dans ses "tableaux poétiques", pour reprendre son expression. Cette appellation témoigne d'un travestissement de la peinture en poésie, en concordance avec l'expérience et la proposition théorique de Butor: "il a fallu que je fasse de la peinture avec mes textes". 
De fait, comme l'a montré Duchamp avec ses ready-made (1913-1914) et ce, bien avant que ne le prévoie Aragon dans son texte de 1930 "La peinture au défi ", cette artiste ne recourt plus à la couleur et à la peinture pour faire naitre des images, mais exploite la forme discursive de la prose, pour la puissance évocatrice et métaphorique du mot, procédé qu'elle combine au collage de fragments poétiques qui marquent la culture littéraire québécoise - dans leur dé-contextualisation ceux-ci constituent, en quelque sorte, des ready-made poétiques -, de même qu'à la représentation visuelle et schématique d'objets issus de la culture artisanale des femmes - ici des mouchoirs cousus et brodés et/ou à celle de formes géométriques simples, comme la spirale.

Par exemple, dans le cas de la série Tourments, Nathalie Caron présente quatre tableaux poétiques construits à partir de la référence aux poésies de Saint-Denys Garneau, Alfred DesRochers, Rina Lasnier et Marie Uguay; ils symbolisent des mouchoirs d'adieu rendant hommage à la mémoire des poètes disparus et illustrent aussi la mémoire des moments intimistes vécus par l'artiste. Ainsi, sur des fonds quadrillés par l'intervention de la couture, on peut voir d'abord l'image sémiotique du mouchoir et lire ensuite, dans une gymnastique du corps et de l'œil, des fragments de poèmes imprimés, disposés linéairement et provenant de la production des poètes identifiés plus haut, auxquels se superposent les courtes propositions lyriques écrites et/ou dessinées par Nathalie Caron et brodées en noir sous la forme iconique de la spirale ${ }^{23}$.

L'hétérogénéité et/ou la diversité des stratégies d'énonciation syntaxiques utilisées ici atteint son paroxysme et adresse, comme dans le cas du ready-made et du collage, un commentaire critique à l'endroit des classifications de genres imposées par la tradition de la spécificité. Faire voir une image, des images, sans faire appel aux moyens de la peinture et pourtant, malgré

23 Les inscriptions littéraires de Nathalie Caron connotent soit le rapport au passé, à l'angoisse, au corps, à la nature; par exemple:

: Lorsque le hurlement s'évanouissait, son regard fouillait l'horizon de brume. Rien n'arrivait comme prévu *.

*Une brise tiède s'élevait et chassait peu à peu les inquiétudes puissantes et dommageables .

- Encore frémissant son corps tombait dans un sommeil profond.

- Ses rêves se peuplaient de froissements de feuilles, de grincements de branches d'arbres. 
l'absence de cette référence, ne jamais cesser de parler d'elle. Car, s'il ne s'agit pas de peintures, au sens propre du terme, il s'agit tout de même de "tableaux" poétiques, fixés au mur comme des peintures, dont la force hétérogène des métissages donne lieu à l'émergence iconique d'un mouchoir et d'une infinité d'images mentales potentielles véhiculées par l'entrecroisement linéaire et spiralé des formulations poétiques, contribuant, sur le plan sémantique/symbolique, à la nature profondément polysémique des œuvres.

De plus, cette combinaison hyper-impure des codes linguistiques et visuels déstabilise les modes de perception acquis du regardeur, qui ne peut plus lire ces ensembles sémiotiques dans une perspective uniquement linéaire (soit de gauche à droite), de même qu'il lui est impossible de capter la totalité de ces textesicônes à partir des codes perceptuels relatifs à l'espace euclidien. Comme dans le cas de la peinture abstraite, le parcours visuel des œuvres hybrides de Nathalie Caron oblige le regardeur à poser une pluralité de regards discontinus, puisque la construction topologique de celles-ci s'affirme comme une forme globale où toutes les régions sont en interaction.

Somme toute, si ce type d'organisation spatiale hétérogène a des rapports d'affinité avec le ready-made, le collage, la poésie, la broderie et la couture, il participe aussi des stratégies d'énonciation propres à la peinture. Ainsi, outre le fait que l'usage du mot est envisagé dans sa potentialité symbolique/iconique, la construction syntaxique de ces textes-icônes se présente physiquement comme une peinture peuplée de signes et de textures qui, par l'effet de tapisserie - suggéré par la distribution à répétition des caractères alphabétiques imprimés -, pourrait faire allusion au all-over et, par la forme identifiable de la spirale, rappeler sa parenté avec les préoccupations de l'abstraction géométrique.

\section{L'hétérogénéité ou le jeu inventif et déréglé du recyclage.}

Comme l'indique l'analyse de ces trois corpus, le phénomène de l'hétérogénéité artistique, que j'associe à l'attitude "postmoderne" et que j'oppose au culte de la spécificité, implique toujours déjà des stratégies de recyclage et de récupération. D'une certaine manière, le recours à l'hétérogénéité permettrait de "faire du neuf avec du vieux" en misant sur la re-création et la 
réorganisation trans-linguistique des codes linguistiques établis par la tradition de la spécificité. Vue ainsi, l'hétérogénéité confronte l'artiste à l'invention et à la production de surprises syntaxiques qui dirigent le contenu sémantique/symbolique des œuvres visuelles "surréalistes " qu'elle engendre dans l'axe polysémique du second degré. C'est, tout au moins, ce que révèlent Duchamp, en récupérant l'objet arbitraire et en le recyclant dans le contexte de l'art, Max Ernst, avec l'usage de l'imprimé et de la photographie dans le collage, et Nathalie Caron, par la reprise, dans ses poésies visuelles, des procédés du ready-made et du collage, ainsi que par l'appropriation des techniques artisanales de la couture et de la broderie. Dans ce sens, la logique "postmoderne" de l'hétérogénéité serait à comprendre dans une perspective ludique, la perspective d'un jeu dont le principal enjeu consiste à détourner toutes les règles, toutes les lois, au profit de l'affirmation libre et nomade du transcodage. Partant de là, le voyage à travers l'histoire des codes artistiques et/ou non artistiques permettrait la célébration de tous les déplacements trans-linguistiques possibles, soit la réapparition de la magie. Car l'art aurait-il une autre fonction que de nous faire pénétrer dans l'univers pulsionnel et symbolique du rêve, là où il n'y a plus de cartes pour diriger nos errances, là où se perdre signifie trouver la voie intérieure du désir, là où le "réel " apparaît comme une fiction? 